\title{
Status of Topotecan in Small-Cell Lung Cancer
}

\author{
P. Drings ${ }^{a} \quad$ R. Stahel ${ }^{b}$ \\ aThoraxklinik Heidelberg gGmbH, bLabor für Onkologie, Departement Innere Medizin, Universitätsspital Zürich
}

The presentations of the symposium 'Revisiting Response in SCLC: Advantage with Topotecan?' (Zürich, April 16.-18., 1999) confirm that topotecan belongs to the most potent substances for this tumor entity. The cytostatic drug has been intensively investigated during the last view years. Remission rates in previously untreated patients with extensive disease reach $40 \%$ and the median survival time is 10 month. Also in pretreated patients the drug proved its efficacy. In cases with remissions after primary therapy, the remission rate in secondline treatment was $38 \%$, whereas it was only $6.4 \%$ in primarily refractory patients.

In the randomized comparison within a phase III study, topotecan proved to be equipotent in the treatment of relapsed patients as a monotherapy compared with the combination of cyclophosphamide, doxorubicin and vincristine $(\mathrm{CAV})$ with respect to remission rates and survival time, and it was even slightly superior with respect to symptom control.
In the meantime, as a logical consequence of its efficiency in the second-line treatment, topotecan has also been used as first-line therapy of small-cell lung cancer. Several studies in the USA and in Germany document the high activity of topotecan in combination with paclitaxel, etoposide or cisplatinum. These studies are currently underway and await final conclusions.

Also interesting is the observation that brain metastases are diminished with this therapy. Possibly, topotecan may develop a protective effect against the clinical manifestation of brain metastasis. In conclusion, topotecan belongs to the most important and inevitable drugs in the treatment of small-cell lung cancer. Up to date, however, its position is only settled beyond any doubt in the second-line treatment. Treatment of the relapse in these patients is frequently difficult and problematic. In this situation, drugs with a good efficacy profile and low side effects are of special advantage.

\section{KARGER}

(c) 2000 S. Karger GmbH, Freiburg

Fax +497614520714 